\title{
LEAD DISCOVERY
}

\section{Screening by signature}

DOI:

$10.1038 / \mathrm{nrd} 2340$
Tumour-specific mutations in cancer present unique targets for highly specific molecularly targeted drugs. However, many of these targets are considered 'undruggable' as they belong to classes of oncoproteins (for example, transcription factors) where traditional high-throughput screening is likely to fail. Reporting in PLoS Medicine, Todd Golub's group now present the first oncoprotein-modulating candidate drug identified by a novel approach - the screening of small-molecule libraries for gene-expression signatures that are indicative of oncoprotein inactivation.

The paediatric tumour Ewing sarcoma is an example of a tumour with a molecularly defined tumourspecific genetic lesion - more than $80 \%$ of patients express the oncogenic EWS/FLI fusion protein. Currently, Ewing sarcoma is treated with combinations of non-targeted cytotoxic agents, and the prognosis for patients with metastatic disease is bleak. Drug development efforts are further hampered by the historical reluctance to test new drugs in children.

The authors propose that drugs for the treatment of Ewing sarcoma might already exist, and performed gene-expression-based highthroughput screening (GE-HTS) on a library of small molecules enriched for FDA-approved drugs. The power of this approach lies in the use of multigene signatures that represent changes of the complex biological state of a cell in response to 'switching off the oncogene.' To identify a EWS/FLI signature, the authors performed genome-wide expression profiling of a Ewing sarcoma cell line with or without inactivation of EWS/FLI by RNA interference. Fourteen genes displayed a statistical expression difference in response to oncogene inactivation, as well as reversion of the expression pattern when a EWS/FLI rescue construct was introduced into the knockdown cells. Together with 2 control genes, the 16-gene signature was converted to a low-cost, high-thoughput format based on ligation-mediated amplification and multicolour-bead-based detection.

Screening a library of more than 1,000 small molecules for the EWS/ FLI signature, cytosine arabinoside (ARA-C), a nucleoside analogue used in the treatment of leukaemia, emerged as the top-scoring candidate. Importantly, it did not induce a 'pseudo' EWS/FLI off signature in ARA-C responsive, EWS/FLI negative acute lymphoblastic leukaemia cells, indicating that its signature is specific in the context of EWS/FLI oncogene expression. Furthermore, ARA-C was shown to induce wholegenome changes that are consistent with EWS/FLI knockdown, and specifically decreased EWS/FLI protein levels. In multiple Ewing sarcoma cell lines, ARA-C was shown to abrogate anchorage-independent growth and inhibit cell viability, and

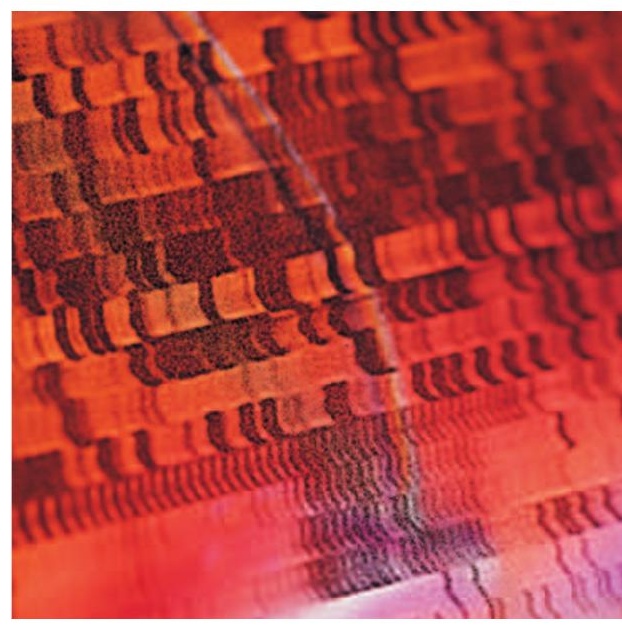

in vivo results showed the inhibition of tumour growth in mouse Ewing sarcoma xenograft models. The mechanism by which it regulates EWS/FLI protein levels remains to be established; however, because ARA-C has a well-characterized safety profile even for paediatric use, the results strongly support the initiation of clinical trials that test ARA-C in children with Ewing sarcoma.

This study demonstrates that GE-HTS constitutes a powerful discovery platform for small-molecule modulators of previously considered 'undruggable' oncogenes. Furthermore, by using screening libraries that are highly enriched for approved drugs, it can provide a rapid path to clinical application. Alexandra Flemming

ORIGINAL RESEARCH PAPER Stegmaier, K. et al. Signature-based small molecule screening identifies cytosine arabinoside as an EWS/FLI modulator in Ewing sarcoma. PLoS Med. 4, e122 (2007)

FURTHER READING Lamb, J. et al. The Connectivity Map: using gene-expression signatures to connect small molecules, genes, and disease. Science 313, 1929-1935 (2006) 